\title{
Evaluation of reservoir connectivity using whole-oil gas chromatographic fingerprint technology: A case study from the $\mathrm{Es}_{3}{ }^{3}$ reservoir in the Nanpu Sag, China
}

\author{
Xu Yaohui ${ }^{1,2 *}$, Shen Xianda ${ }^{1}$, Chen Nengxue ${ }^{3}$, Yang Cuimin ${ }^{3}$ and Wang \\ Qiaoli $^{4}$ \\ ${ }^{1}$ Department of Geochemistry, Yangtze University, Hubei 434023, China \\ ${ }^{2}$ Shandong Provincial Key Laboratory of Depositional Mineralization \& Sedimentary Minerals, Shandong University of \\ Science and Technology, Shandong 266510, China \\ ${ }^{3}$ Research Institute of Petroleum Exploration and Development, CNPC Jidong Oilfield, Hebei 063000, China \\ ${ }^{4}$ State Key Laboratory of Petroleum Resources and Prospecting, China University of Petroleum, Beijing 102249, China \\ - China University of Petroleum (Beijing) and Springer-Verlag Berlin Heidelberg 2012
}

\begin{abstract}
In this study, whole-oil gas chromatographic fingerprint analyses were performed on oils from the $\mathrm{Es}_{3}{ }_{3}^{3}$ reservoir in the Liubei area of the Nanpu Sag. The gas chromatographic peaks of cyclic and branched alkanes with relatively high resolution from $n \mathrm{C}_{10}$ to $n \mathrm{C}_{25}$ were selected to establish a database of whole-oil gas chromatographic peak height ratio fingerprints. Reservoir fluid connectivity was identified by using clustering analysis. This method can reflect the gas chromatography fingerprint information accurately and entirely, and avoid the one-sidedness of the star diagram method which only selects several fixed gas chromatographic peaks.
\end{abstract}

Key words: Whole-oil gas chromatographic fingerprint technology, reservoir connectivity, Nanpu Sag, $\mathrm{Es}_{3}{ }^{3}$ reservoir, clustering analysis

\section{Introduction}

The identification of compartments and connectivity of reservoirs is an important aspect of hydrocarbon reservoir evaluation, which can provide relevant information for oilfield development and establishment of production programs (Hwang et al, 1994; Peters and Fowler, 2002). Although the reservoir geochemical method is quick, simple and low-cost for evaluating the fluid connectivity in reservoir and the whole-oil gas chromatographic (GC) fingerprint analysis used for studying reservoir connectivity was first reported in 1994, this method has not been extensively used in petroleum companies. The reason was that the method can only be applied to a few samples. The whole oil GC fingerprint technology is based on the fact that the whole oil GC fingerprint characteristics of the oils from different reservoirs or from a separated reservoir that is formed by facies change have obvious differences, whereas oils from connected reservoirs show consistent whole oil GC fingerprint characteristics (Hwang et al, 1994; Baskin and Hwang, 1995; Kaufman et al, 2002; Peters and Fowler, 2002). In this method, the oil and each component are analyzed by GC, and some paired hydrocarbons were selected from the whole oil

* Corresponding author. email: yaohuixu@126.com

Received August 20, 2011
GC. The relative compositions of every pair of compounds (the ratio of peak areas or peak heights from adjacent peaks or close peaks) were calculated, and a star diagram using polar coordinates that characterizes the GC fingerprint parameters was drawn, so the oil group can be distinguished and reservoir connectivity can be identified (Halpern, 1995; Wever, 2000; He et al, 2001; 2004; Huang et al, 2002; Jin et al, 2009). The star diagram method is only suitable for comparing the GC fingerprints of oil from a few wells.

The $\mathrm{Es}_{3}{ }^{3}$ reservoir in the Liubei area of the Nanpu Sag is discussed in this paper. Instead of selecting several relevant hydrocarbon compounds from $\mathrm{GC}$ as described in previous papers, we selected the paired GC peaks of cyclic and branched alkanes with relatively high resolution from $n \mathrm{C}_{10}$ to $n \mathrm{C}_{25}$. The ratios of peak areas or peak heights of every pair of molecules were calculated as the relative composition, so we can obtain hundreds of pairs of ratio data. All the fingerprint data of each oil sample were taken as one sample. A clustering analysis method was employed to determine the similarity and differences of samples, and to identify reservoir connectivity. This method can accurately reflect the whole oil GC fingerprint information.

\section{Samples and geological setting}

$\mathrm{The} \mathrm{Es}_{3}{ }^{3}$ reservoir in the Liubei area of the Nanpu Sag was formed on a fault nose structure and is dominated by 
formation-structure traps and fault-structure traps, with a closed area of about $2.5-5 \mathrm{~km}^{2}$ and oil reserves over 10 million tonnes. The reservoir has medium porosity and permeability, and the crude oil physical properties are good (Table 1), with low density $\left(0.8535 \mathrm{~g} / \mathrm{cm}^{3}\right)$, low viscosity $(7.66 \mathrm{mPa} \cdot \mathrm{s})$, high wax content $(15.9 \%)$ and high solidifying point $\left(31.7^{\circ} \mathrm{C}\right)$. The crude oil in the $\mathrm{Es}_{3}{ }^{3}$ reservoir in the Liubei area has the same source rocks and similar filling history. In plan view, the $\mathrm{Es}_{3}{ }^{3}$ reservoir consists of three parallel fan delta sand bodies that are NE-SW oriented, and in vertical direction the reservoir can be divided into five oil groups: II, III, IV, $\mathrm{V}$ and $\mathrm{Es}_{3}{ }^{5}$. The III-V oil groups have a consistent oil-water interface (about $3250 \mathrm{~m}$ ), and the IV oil group is the most developed. The high heterogeneity of sand layers of each oil group and the difference in human factors lead to some problems such as the consistency in oil group division and reservoir correlation and connectivity evaluation of adjacent production wells in the $\mathrm{Es}_{3}{ }^{3}$ reservoir.

Table 1 Oil properties characteristics from typical production wells

\begin{tabular}{cccccccc}
\hline Well & Horizon & Depth, $\mathrm{m}$ & Density, $\mathrm{g} / \mathrm{cm}^{3}$ & Viscosity $50^{\circ} \mathrm{C}, \mathrm{mPa} \cdot \mathrm{s}$ & Solidifying point, ${ }^{\circ} \mathrm{C}$ Sulphur content, \% & Wax content, \% \\
\hline LB1-15-20 & $\mathrm{Es}^{3}{ }^{3}$ & $2918.4-2942.0$ & 0.8458 & 6.69 & 29.0 & 0.07 & 11.89 \\
L13-19 & $\mathrm{Es}_{3}{ }^{3}$ & $3154.0-3221.0$ & 0.8604 & 0.014 & 34.5 & 0.02 & 17.73 \\
L15-24 & $\mathrm{Es}_{3}{ }^{3}$ & $3015.0-3076.6$ & 0.8518 & 9.58 & 34.1 & 0.12 & 20.90 \\
L15-16 & $\mathrm{Es}_{3}{ }^{3}$ & $3062.8-3174.0$ & 0.85634 & 14.376 & 29.0 & 0.05 & 13.06 \\
& Average & 0.8535 & 7.66 & 31.7 & 0.07 & 15.90 \\
\hline
\end{tabular}

In this study, we collected oil samples from 16 wells in the principal part of the reservoir. The whole oil GC fingerprint analyses were performed on oil samples with an Aglient GC6890N using an HP-5 quartz capillary column $(30 \mathrm{~m} \times$ $0.25 \mathrm{~mm} \times 0.25 \mu \mathrm{m})$, with a flow velocity of $0.7 \mathrm{~mL} / \mathrm{min}$. The temperature program was as follows: initially the temperature was set at $50^{\circ} \mathrm{C}$ for $1 \mathrm{~min}$, next it was increased to $100^{\circ} \mathrm{C}$ at a rate of $20^{\circ} \mathrm{C} / \mathrm{min}$, then it was increased to $290^{\circ} \mathrm{C}$ at a rate of $5^{\circ} \mathrm{C} / \mathrm{min}$ and held for $20 \mathrm{~min}$.

The comparison of the measured concentration of compounds between two repeated GC analyses and calculation based on the same oil from well LB1-4 shows that the repeatability is very good (Fig. 1).

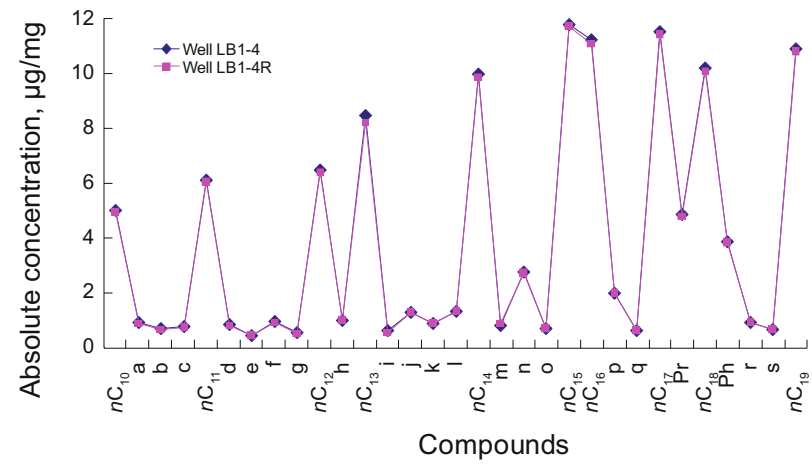

Fig. 1 Comparison of the measured concentration of compounds between two repeated GC analysis and calculation. a, b, c, .., s represent cyclic and branched alkanes ranging from $n \mathrm{C}_{10}$ to $n \mathrm{C}_{19}$

\section{Establishment of the whole oil GC fingerprint parameter database}

In the whole oil GC graph, the peaks that have relatively low abundance and lie between peaks of $n$-alkanes represent cyclic and branched alkanes. In general, cyclic and branched alkanes have more stable chemical properties than $n$-alkanes, and their abundance distributions may be used to construct the GC fingerprint characteristics of crude oil chemical composition. In order to obtain the fingerprint database, paired peaks of cyclic and branched alkanes ranging from $n \mathrm{C}_{10}$ to $n \mathrm{C}_{25}$ were selected and numbered. For example, the whole oil GC of well L17-21 was analyzed, and 159 peaks were marked out (Fig. 2). The heights of these peaks were calculated to establish the original peak height database of whole oil GC of all oil samples (Table 2). We only list part of the peak height data of the oil samples from six wells.
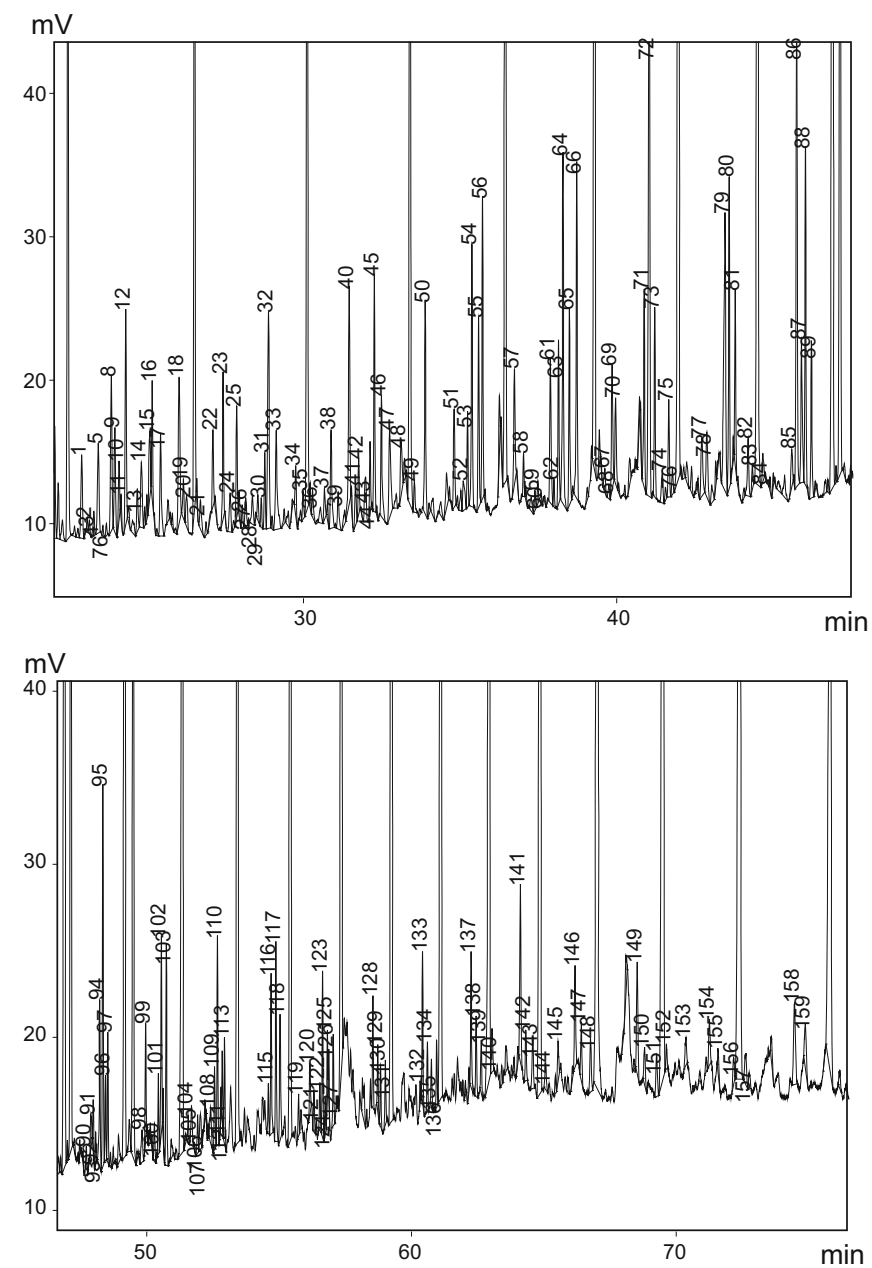

Fig. 2 Numbered peaks of cyclic and branched alkanes in the whole oil GC of well L17-21 
Table 2 The original peak height database of the whole oil GC

\begin{tabular}{ccccccc}
\hline Well & LB1-9 & LB1-7 & L9-15 & L9-12 & L17-21 & LB1-13 \\
\hline 1 & 9084 & 6768 & 10841 & 10586 & 5764 & 7453 \\
\hline 2 & 1558 & 1100 & 1787 & 1567 & 924 & 1233 \\
3 & 1633 & 972 & 1642 & 1627 & 896 & 1200 \\
4 & 2934 & 1954 & 3159 & 3357 & 1704 & 2088 \\
5 & 9582 & 7596 & 12811 & 12039 & 6282 & 7963 \\
6 & 1246 & 822 & 1402 & 1578 & 739 & 871 \\
7 & 1300 & 894 & 1543 & 1241 & 848 & 1065 \\
8 & 17637 & 12762 & 20144 & 20479 & 10802 & 13832 \\
9 & 11175 & 9545 & 14786 & 15988 & 7266 & 9446 \\
10 & 6513 & 5365 & 7789 & 7743 & 4160 & 5559 \\
155 & 1972 & 1247 & 2572 & 3983 & 1274 & 1686 \\
156 & 1853 & 2056 & 2937 & 1277 & 1512 & 1594 \\
157 & 1722 & 2261 & 2343 & 3422 & 1828 & 1796 \\
\hline 158 & 5230 & 6069 & 6496 & 7313 & 4742 & 5179 \\
\hline 59 & 3347 & 5945 & 5515 & 4258 & 2560 & 3596 \\
\hline 1,3 & $\ldots 158$, & & & & & \\
\hline
\end{tabular}

Notes: 1, 2, 3.. 158, 159 represent the peak numbers in Fig. 2

Based on this original peak height database, every peak height was divided by the next four peak heights respectively and the GC fingerprint peak height ratio database was established (Table 3). We only list part of the peak height ratio data of the oil samples. This database was used to identify reservoir connectivity in the following experiment.

Table 3 The whole oil GC fingerprint peak height ratio database

\begin{tabular}{|c|c|c|c|c|c|c|}
\hline $\begin{array}{l}\text { Fingerprint } \\
\text { parameters } \\
\text { (peak height ratio) }\end{array}$ & LB1-9 & LB1-7 & L9-15 & L9-12 & L17-21 & LB1-13 \\
\hline $1 / 2$ & 5.83 & 6.15 & 6.07 & 6.76 & 6.24 & 6.04 \\
\hline $1 / 3$ & 5.56 & 6.96 & 6.60 & 6.51 & 6.43 & 6.21 \\
\hline $1 / 4$ & 3.10 & 3.46 & 3.43 & 3.15 & 3.38 & 3.57 \\
\hline $1 / 5$ & 0.95 & 0.89 & 0.85 & 0.88 & 0.92 & 0.94 \\
\hline $2 / 3$ & 0.95 & 1.13 & 1.09 & 0.96 & 1.03 & 1.03 \\
\hline $2 / 4$ & 0.53 & 0.56 & 0.57 & 0.47 & 0.54 & 0.59 \\
\hline $2 / 5$ & 0.16 & 0.14 & 0.14 & 0.13 & 0.15 & 0.15 \\
\hline $2 / 6$ & 1.25 & 1.34 & 1.27 & 0.99 & 1.25 & 1.42 \\
\hline $3 / 4$ & 0.56 & 0.50 & 0.52 & 0.48 & 0.53 & 0.57 \\
\hline $3 / 5$ & 0.17 & 0.13 & 0.13 & 0.14 & 0.14 & 0.15 \\
\hline $3 / 6$ & 1.31 & 1.18 & 1.17 & 1.03 & 1.21 & 1.38 \\
\hline $3 / 7$ & 1.26 & 1.09 & 1.06 & 1.31 & 1.06 & 1.13 \\
\hline $4 / 5$ & 0.31 & 0.26 & 0.25 & 0.28 & 0.27 & 0.26 \\
\hline $156 / 158$ & 0.35 & 0.34 & 0.45 & 0.17 & 0.32 & 0.31 \\
\hline $156 / 159$ & 0.55 & 0.35 & 0.53 & 0.30 & 0.59 & 0.44 \\
\hline $157 / 158$ & 0.33 & 0.37 & 0.36 & 0.47 & 0.39 & 0.35 \\
\hline $157 / 159$ & 0.51 & 0.38 & 0.42 & 0.80 & 0.71 & 0.50 \\
\hline $158 / 159$ & 1.56 & 1.02 & 1.18 & 1.72 & 1.85 & 1.44 \\
\hline
\end{tabular}

\section{Identifying reservoir connectivity using the whole oil GC fingerprint database}

In this study, the GC fingerprint analyses were performed on oils from 16 wells of the $\mathrm{Es}_{3}{ }^{3}$ reservoir in the Liubei area of the Nanpu Sag. According to the above described method, the whole oil GC fingerprint parameter database of the 16 wells was established (Table 3 ), and clustering analysis was performed on this basis (Fig. 3).

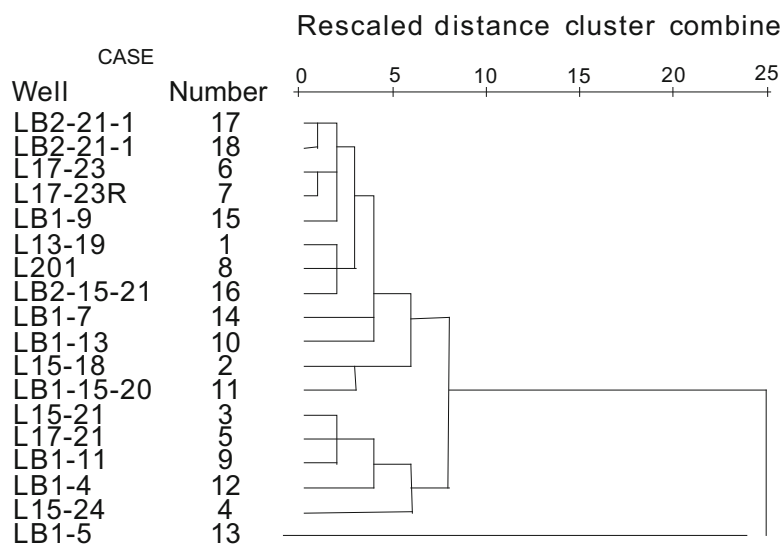

Fig. 3 Clustering tree diagram of whole oil GC fingerprint parameter from 16 wells of $\mathrm{Es}_{3}{ }^{3}$ reservoir in the Liubei area

As shown in Fig. 3, the two LB2-21-1 groups have identical GC fingerprint parameter data. L17-23 and L1723R groups are the GC fingerprint parameter data of one oil sample obtained from repeated measurements. The nearest groups are the two LB2-21-1 groups, and the L17-23 and L17-23R groups from repeated measurements, indicating good repeatability of the whole oil GC analysis.

In addition, there are three close well groups: L17-23, LB2-21-1 and LB1-9; L13-19, L201 and LB2-15-21; L15$21, \mathrm{~L} 17-21$ and LB1-11. It is believed that the GC fingerprint parameters of the above three well groups are very similar, indicating the excellent reservoir connectivity. The clustering analysis of other well groups indicates that the distance of the well groups is relatively great, and the reservoir connectivity is poor. Fig. 4 shows the reservoir connectivity in the Liubei $\mathrm{Es}_{3}{ }^{3}$ reservoir.

\section{Discussion}

The reservoir correlation and oil production test results of well LB1-15-20, LB2-15-21, L13-19 and L201 are shown in Fig. 5. It is clear that the results of well LB2-15-21, L13-19 and L201 are in accordance with the GC fingerprint analysis results, namely, the production layers of the three wells have good connectivity. According to the reservoir correlation of well LB1-15-20 and LB2-15-21, the two wells have good reservoir connectivity, while the GC fingerprint analysis results show that they have poor reservoir connectivity (Fig. 3 ). It is seemed that the conclusions from the two methods are contradictory. Although well LB1-15-20 and LB2-15-21 have oil layers that are well-connected, the two wells have different oil production layers. The production layers of LB1-15-20 


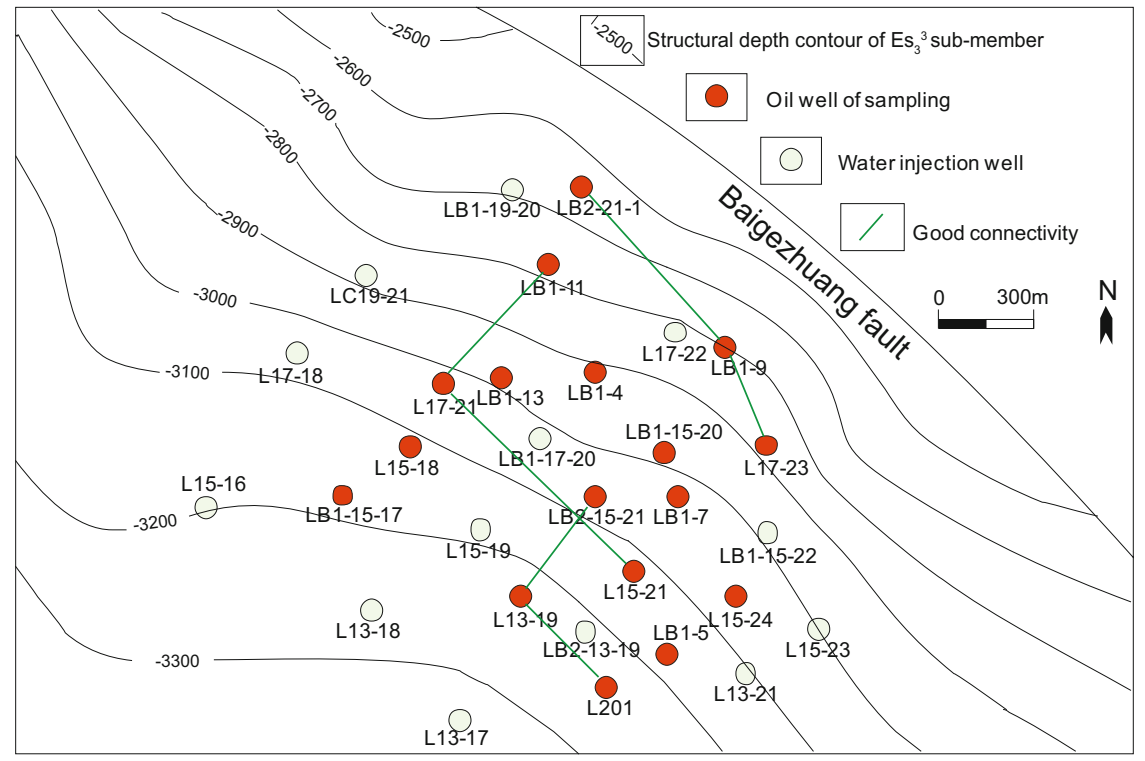

Fig. 4 Schematic diagram of reservoir connectivity of $\mathrm{Es}_{3}{ }^{3}$ reservoir

are above the connected oil layer and those of LB2-15-21 are under the connected oil layer. Thus the oil of the two wells is not completely from the connected oil layer, which causes the different GC fingerprint results, namely, the clustering analysis indicates that the distance was relatively far, and the reservoir connectivity was poor.

The well-connecting section through well LB1-9 and L1723 was studied. Before reservoir connectivity evaluation, the reservoirs of well LB1-9 and L17-23 were considered to be uncorrelated and the oil production layers were different. However, well LB1-9 and L17-23 have very similar whole oil GC fingerprint analysis results (Fig. 3). In addition, it can be seen from the production data that, the daily production of well LB1-9 and L17-23 in March 2009 was 2.20 and 1.91 tons respectively, which was very close, indicating the excellent reservoir connectivity between the two wells. Combined with logging data, it is believed that the production layers of well LB1-9 and L17-23 are the same and they have good reservoir connectivity (Fig. 6).

\section{Conclusions}

In the study, the GC fingerprint peak height ratio database was established based on the GC peaks of cyclic and branched alkanes with relatively high resolution from $n \mathrm{C}_{10}$ to $n \mathrm{C}_{25}$. All the fingerprint data of every oil sample were taken as one sample. Reservoir connectivity was identified by using

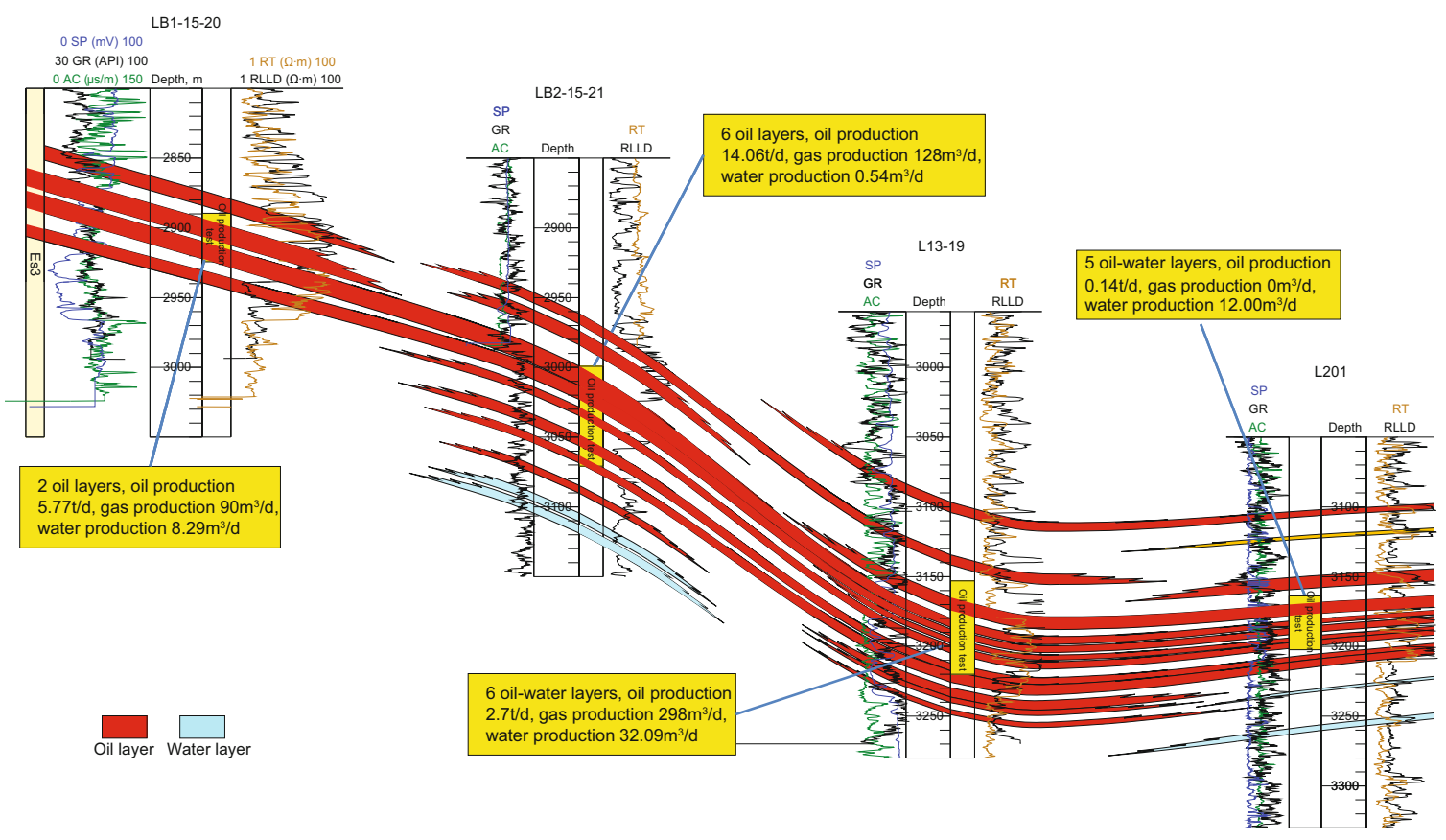

Fig. 5 Reservoir correlation profiles of well LB1-15-20, LB2-15-21, L13-19 and L201 


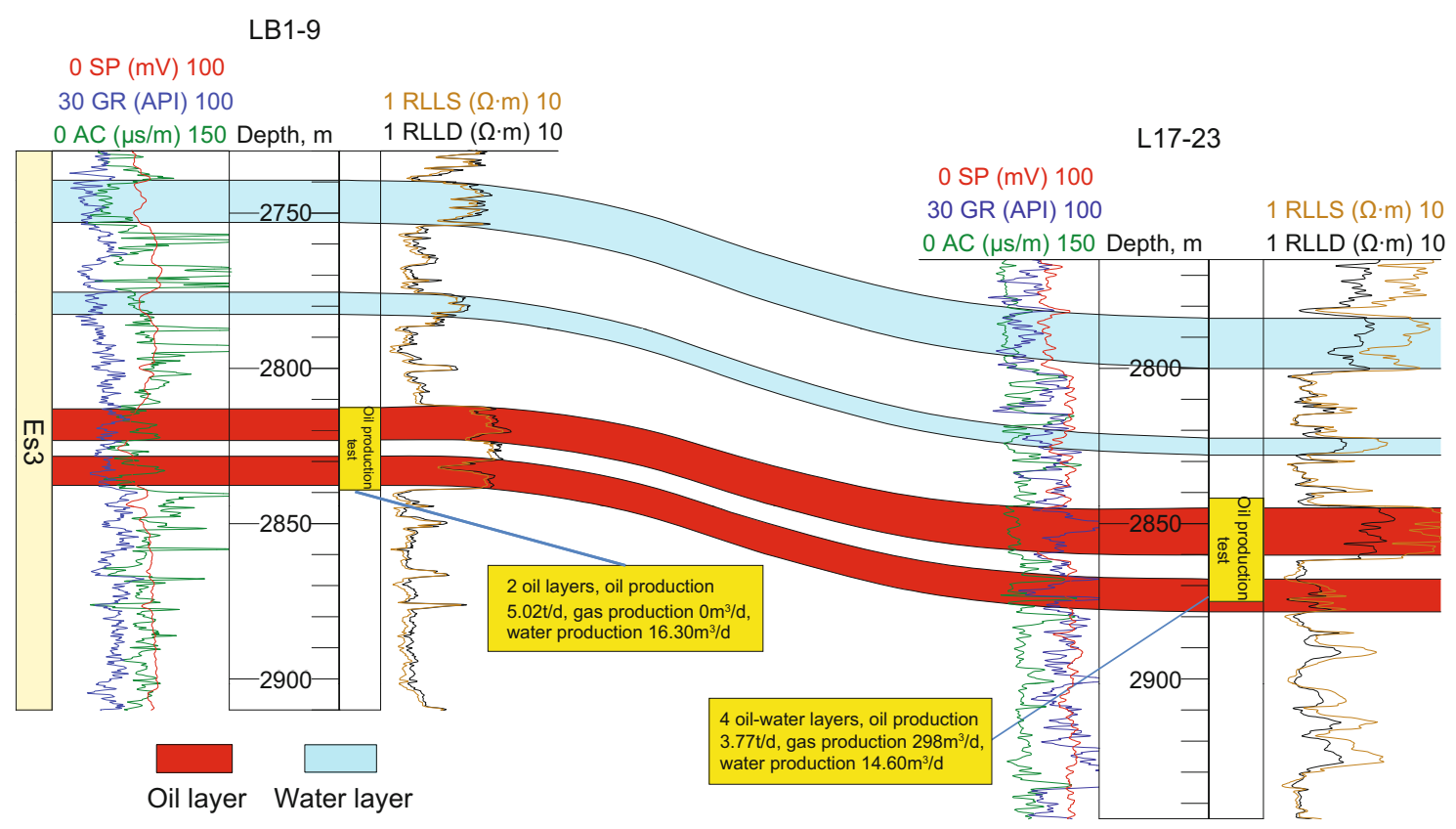

Fig. 6 Reservoir correlation profiles of wells LB1-9 and L17-23 after evaluation of reservoir connectivity

clustering analysis. Results suggested that this method can reflect the whole oil GC fingerprint information accurately and avoid the one-sidedness of the star diagram method. According to the above analysis of the well groups, the whole oil GC fingerprint technology should be applied combined with practical production development data such as well logging and oil production test, which makes the reservoir connectivity evaluation closer to the actual conditions. It is an effective supplementary means to review and check the oil layer division and correlation of production wells, and provides the basis for adjusting development plans.

\section{Acknowledgements}

This work has been funded by Shandong Provincial Key Laboratory of Depositional Mineralization \& Sedimentary Minerals (Project DMSM201009) and Key Laboratory of Tectonics and Petroleum Resources (China University of Geosciences), Ministry of Education, China (Project TPR2010-29). The authors are grateful to Professor Tieguan Wang from China University of Petroleum (Beijing) and Professor Peirong Wang from Yangtze University for their assistance.

\section{References}

Baskin D K and Hwang R J. Predicting gas, oil, and water intervals in Niger Delta Reservoirs using gas chromatography. AAPG Bulletin. 1995. 79: 337-350

Halpern H I. Development and applications of light-hydrocarbon-based star diagrams. AAPG Bulletin. 1995. 79: 801-815

He W X, Wang P R, Liu Y, et al. Proportioning fingerprinting plate of single-source oil samples and its application to the analysis of commingled well oils. Petroleum Exploration and Development. 2001. 28(6): 82-83 (in Chinese)

He W X, Wu S H, Gong H Q, et al. A new method for quantitative identification of fluid continuity in reservoir. Acta Petrolei Sinica. 2004. 25(6): 78-82 (in Chinese)

Huang B J, Li X H and Chen F X. An application of geochemical fingerprint techniques to insight into reservoir communication and production allocation: A case study from WZ12-1 oil field and DF11 gas field. China Offshore Oil and Gas. 2002. 16(5): 302-308 (in Chinese)

Hwang R J, Ahmed A S and Moldowan J M. Oil composition variation and reservoir continuity: Unity field, Sudan. Organic Geochemistry. 1994. 21(2): 171-188

Jin X H, Gang W Z, Lin R Z, et al. Dynamic monitoring of chromatographic fingerprint of reservoir fluids and its application. Oil and Gas Geology. 2009. 30(5): 657-661 (in Chinese)

Kaufman R L, Dashti H, Kabir C S, et al. Characterizing the Greater Burgan field: Use of geochemistry and oil fingerprinting. SPE Reservoir Evaluation \& Engineering. 2002. 5(3): 190-196

Peters K E and Fowler M G. Applications of petroleum geochemistry to exploration and reservoir management. Organic Geochemistry. 2002. 33(1): 5-36

Wever H E. Petroleum and source rock characterization based on $\mathrm{C}_{7}$ star plot results: examples from Egypt. AAPG Bulletin. 2000. 84: 10411054 